TP Periodica Polytechnica Chemical Engineering

61(1), pp. 10-14, 2017

DOI: $10.3311 /$ PPch.10103

Creative Commons Attribution (i)

RESEARCH ARTICLE

\section{Relationship between Commonly Used Adsorption Isotherm Equations Impedes Isotherm Selection}

\author{
Zsanett Dorkó ${ }^{1,2}$, Anett Szakolczai ${ }^{1}$, Blanka Tóth ${ }^{1}$, George Horvai ${ }^{1,2 *}$
}

Received 04 October 2016; accepted after revision 22 December 2016

\begin{abstract}
If the measured isotherm data of an adsorption system are well described by the Freundlich equation, then they can similarly well be described by the bi-Langmuir or tri-Langmuir model in most practical cases. This is proved by Monte Carlo simulation and by comparison of the mathematical functions of the respective isotherm models.
\end{abstract}

\section{Keywords}

isotherm fitting, bi-Langmuir isotherm, Freundlich isotherm, adsorption isotherm, binding site

\section{Introduction}

Adsorption from liquids on solids is the basic phenomenon in various technologies and environmental phenomena and also in liquid chromatography. Adsorption is also important in many sensors (e.g., quartz crystal microbalance sensors) or with novel materials like molecularly imprinted polymers (MIP) [1-3].

Understanding of adsorption phenomena requires molecular level information about the interaction between the adsorbent and the adsorptive. In some cases this information can be obtained directly, e.g., by using surface spectroscopies, atomic force microscopy, etc. In many other cases such direct information is not available. For example polymeric adsorbents are often crosslinked polymers. Their binding sites may have a different local structure from the rest of the polymer, but not sufficiently different to be distinguishable by solid state spectroscopies. Soils represent another type of solids with complex structure where the structure of binding sites may elude exact characterization.

A generic method to study such complex adsorbents is to investigate the adsorption quantitatively, i.e., to study the adsorbed quantity as a function of the equilibrium solution (or gas) concentration of the adsorptive. The observed empirical relationship between the adsorbed $(q)$ and the solution concentrations $(c)$ at a given temperature is the equilibrium isotherm. Isotherms may be studied and compared at different temperatures or with different adsorptives. If more than one compound is simultaneously adsorbed the isotherm is often called competitive isotherm, although simultaneous adsorption may also be synergistic [4].

Measured isotherms consist of individual points. If a curve is fitted to these points it may be used for interpolation. However, the goal of fitting a mathematical function to the measured isotherm points may serve also the goal of discovering some details about the chemistry of binding. This is achieved by the fitting of mathematical functions which had been derived for some hypothetical binding chemistries. Many models and equations have been proposed to describe adsorption in various systems [5-8]. Some popular isotherm equations are the Langmuir, the bi-Langmuir, the Freundlich and the Toth isotherms [9], but many others are also routinely being tested. 
Sometimes such model equations are used merely as interpolation functions, but often the good fit to the experimental points is interpreted as a proof for the underlying model to be applicable to the investigated adsorption system. If this is done, one should check if alternative, feasible model equations do not give similarly good fit to the measured data.

It has often been observed, however, that one can fit different types of isotherm equations to the same experimental data with nearly equal success [10-23]. In such case one may try to improve the measurement precision so that a clear distinction be possible. But increasing the measurement precision is generally expensive in terms of time and equipment. Therefore it is of importance to be able to estimate the necessary minimum of precision which is enough for the safe distinction between the likely models.

The goal of this work is to find out what measurement precision is required to distinguish between the Freundlich isotherm and members of the Langmuir isotherm family, i.e., the Langmuir, bi-Langmuir, tri-Langmuir, etc. isotherms. It has indeed often been observed with imprinted polymers and with soils that the Freundlich isotherm gave a good fit to the measured isotherm data, but the fit with the bi-Langmuir or tri-Langmuir isotherm was similarly good.

It will be shown, that if the Freundlich isotherm gives good fit in the measured concentration range then it is very difficult to reject the bi-Langmuir or the tri-Langmuir model based solely on that single isotherm data series. Extending the investigated concentration range within reasonable limits (but still in the range of Freundlich behaviour) does not lead out from this problem. Note, however, that if experimental measurements are possible below or above the range of Freundlich type behaviour, one may get more information about the adsorption equilibrium.

\section{The Freundlich isotherm and the Langmuir isotherms}

The Freundlich isotherm equation is:

$$
q=a c^{m}
$$

where $q$ is the equilibrium adsorptive concentration in the solid phase, $c$ is the equilibrium adsorptive concentration in the liquid phase, and $a$ and $m$ are constant parameters, $a>0$ and $0<m<1$. If $m=1$ the isotherm is linear. (Note that many authors denote the Freundlich exponent as $1 / n$, where $n>1$.)

The bi-Langmuir equation is:

$$
q=\left(a_{1} c /\left(1+b_{1} c\right)\right)+\left(a_{2} c /\left(1+b_{2} c\right)\right)
$$

where $a_{1}, a_{2}, b_{1}$ and $b_{2}$ are constant parameters. Eq. (2) is used to describe a sorbent with two types of independent binding sites. The concentration of the two sites in the solid phase is $q_{\text {site }, 1}=a_{1} / b_{1}$ and $q_{\text {site }, 2}=a_{2} / b_{2}$, respectively. The binding equilibrium constants of the two sites are $b_{1}$ and $b_{2}$, respectively. The Langmuir isotherm contains only the first additive term of the right hand side of Eq. (2), the tri-Langmuir isotherm consists of three additive terms.

\section{Monte Carlo simulation}

A simple numerical simulation example will show the fallacies of model fitting. It will be assumed that the true model is the Freundlich model and "experimental" data will be simulated by the Monte Carlo method. Then both the Freundlich and the bi-Langmuir model will be fitted to the "experimental" data. It will be found that the bi-Langmuir model fits these simulated data better than the Freundlich model.

For the simulation it was assumed that the "true" isotherm of a system is of the Freundlich type and the measurements are superimposed by random experimental errors. The Freundlich isotherm parameters were chosen to be $a=1000$ and $m=0.8$. The random errors of measurement were supposed to be normally distributed with 3\% standard deviation. A Monte Carlo simulation has been carried out with these data to generate 20 simulated isotherm measurements in the concentration range 1 to 100 (in arbitrary concentration units). Seven "measurement" points were logarithmically evenly distributed in this range.

Subsequently the Freundlich isotherm (Eq. (1)) and the bi-Langmuir isotherm (Eq. (2)) were fitted to each of the 20 simulated, error loaded isotherms. In 13 of the 20 simulated experiments the bi-Langmuir equation gave better fit to the data (as evidenced by the lower sum of the squared relative deviations) than the case-by-case best fitting (not the "true") Freundlich equation.

This result shows that in a similar real experiment it would be more likely to find the bi-Langmuir model to be the true isotherm type, although in this case obviously the Freundlich model is the true one. Moreover, the scatter of the parameters of the best fitting bi-Langmuir curves in these simulated parallel experiments was very large. The relative standard deviation of the binding constants and of the site capacities were between $41 \%$ and $79 \%$. In contrast to this, the relative standard deviation of the fitted Freundlich parameters was only $2 \%$ and $1 \%$ for $a$ and $m$, respectively. This means that although the biLangmuir model gave in the majority of the cases the better fit to the simulated data, the bi-Langmuir parameters themselves were rather uncertain.

\section{Comparison of the isotherm functions}

The Monte Carlo simulation had shown for a particular parameter set of the Freundlich isotherm, that model fitting may lead to wrong conclusions about the adequate model describing an experiment. The question is if this observation is generally valid, i.e., if the true isotherm is the Freundlich isotherm, then the bi-Langmuir model will give similar or better fit to the measured data.

This question will be investigated here for a subset of all possible cases. The validity of the Freundlich model will be 
assumed with the Freundlich parameter $a=1000$ and for five values of the $m$ parameter: $0.1,0.3,0.5,0.7$ and 0.9 . The concentration range will be 1 to 1000 (in arbitrary concentration units). Random errors will not be simulated. The idea behind the method used is as follows. If the wrong model can give a good fit to the measured data (which have a moderate random error), than this is a sign that the wrong model is able to approach very closely the mathematical curve described by the good model. Thus the question will be how closely can the biLangmuir model be fitted to the error-free Freundlich curve.

The calculations made are simple. For each of the five Freundlich parameter set mentioned above, the $q$ values were calculated at 31 , logarithmically equidistant $c$ values in the range 1 to 1000 . Then the bi-Langmuir equation was fitted to each of the five point sets. Fitting was done by minimizing the squared maximum relative deviation of the two functions. The Solver function of Microsoft Excel was used with weights inversely proportional to the squared Freundlich $q$ values. The results were also cross-checked with the Wolfram Mathematica software. The best fitting bi-Langmuir parameters were noted.

Fig. 1 shows the deviations of the best fitting bi-Langmuir function curves from their corresponding Freundlich curves as a function of $\log c$. One can see from this figure that the deviations change sign four times and in this way they resemble random deviations. The size of the maximum deviation depends on the Freundlich parameter $m$, and it goes from ca. $2 \%$ (for $m=0.1$ and $m=0.9$ ) to $7 \%$ (for $m=0.5$ ).

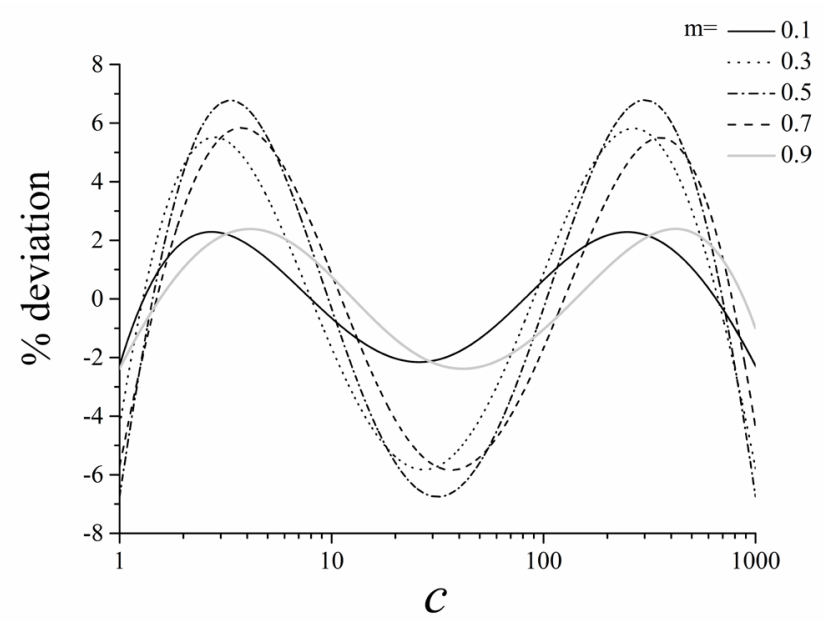

Fig. 1 Deviation of the adsorbed concentrations calculated with the best fitting bi-Langmuir parameters from those calculated using the Freundlich equation with $a=1000$ and the $m$ values shown in the figure

These results mean, that the bi-Langmuir model can indeed quite closely approach the Freundlich isotherm. In the literature one can find many papers where isotherms have been measured with 5-10\% standard deviation [24, 25]. With such precision it appears impossible to decide if isotherm data which can be fitted reasonably with the Freundlich equation are indeed Freundlich type or rather bi-Langmuir type. Further calculations like these have shown that if the concentration range is only two orders of magnitude wide (from 1 to 100), then the maximum deviation between the two models is ca. $2.5 \%$. This maximum error is at $m=0.5$, while at other $m$ values it is considerably less, similarly to Fig. 1.

The tri-Langmuir model gives even better fits to the Freundlich model. Similar calculations to those shown here result in a maximum deviation of $1.1 \%$ at $m=0.5$, even in a three orders of magnitude wide concentration range (1 to 1000). The sign of the deviations changes six times, thus resembling random errors.

\section{Conclusions}

The Monte Carlo simulation and the comparison of isotherm model functions have shown that the bi-Langmuir or the triLangmuir model can very closely fit isotherm data obeying the Freundlich equation. (The discussion in this paper has been limited to the situation when the Freundlich isotherm is valid throughout the studied concentration range. In many instances this range can be substantially wider than three orders of magnitude.) Since today the highest precision achieved in isotherm measurement has about $1 \%$ standard deviation $[19,26]$, it is virtually impossible to reject the bi-Langmuir or the tri-Langmuir model even if measurements are made in a concentration range two or three orders of magnitude wide, respectively. The precision of $1 \%$ is anyway difficult to achieve as it requires the use of HPLC columns packed with the adsorbent. Many adsorbents are unsuitable for HPLC, and therefore their isotherms are measured with less precision, so that isotherm distinction becomes more difficult.

The demonstration of the above results was limited here to the specific concentration ranges 1 to 100 and 1 to 1000 . The Freundlich parameter $a$ was limited to the single value of 1000 . One can show, however, by purely mathematical means, that the results obtained in this paper remain exactly the same if the concentration range is shifted (but its width remains 2 or 3 orders of magnitude, respectively). Similarly the results are invariant to the value of the Freundlich parameter $a$.

As Fig. 1 shows the position of the largest deviations of the Langmuir functions from the Freundlich function is at welldefined parts of the measurement range. This may be utilized for designing the allocation of measurement points. The decisive power of the measurements may be improved somewhat by allocating more points in these regions.

While one can fit the bi-Langmuir or tri-Langmuir isotherm models very closely to Freundlich isotherm data, the reverse is not true, as one can see by simple counterexamples. (For example the simple Langmuir isotherm cannot be well fitted by the Freundlich model if the concentration range includes both the linear and the saturation part. Indeed in the linear part the Freundlich equation would well fit with $m=1$ and on the horizontal part with $m=0$, but these two mutually exclude each other.) 
This observation might be interpreted by the generally held view that with more parameters ( 4 in case of bi-Langmuir and 6 in tri-Langmuir) one can achieve better fits than with less parameters ( 2 for Freundlich). This view is, however, not generally true. A famous case in mathematics has been the difficult approximation of the square root function by polynomials. Note here that the Freundlich function is for $m=0.5$ the square root function. Further on, using more parameters for fitting may result in good fit but poor reproducibility of the fitted parameters in repeated experiments, as had been demonstrated here with the Monte Carlo method.

As noted earlier, the Freundlich isotherm has quite often been found to well describe experimental data. Sometimes this has been interpreted as a sign of a very heterogeneous binding site distribution (with respect to binding energies). Alternatively, the bi- or tri-Langmuir isotherm was fitted to the data and since these models gave also god fit (not surprisingly in view of the present paper), these latter models were accepted because they are more simply interpreted physically. Both approaches rely on the assumption that the adsorbent has 2, 3 or many Langmuir type binding sites. It is worth noting, however, that if the binding reaction is not of the type

$$
\mathrm{A}+\mathrm{B}=\mathrm{AB}
$$

as assumed in the langmuirian models, but for example of the type

$$
\mathrm{A}+\mathrm{B}=\mathrm{C}+\mathrm{D}
$$

(like in ion exchange), then the adsorption isotherm equation is different from the Langmuir equation even if there exists only one type of site on/in the adsorbent. Under certain quite practical conditions one may obtain for such adsorption sites an isotherm equation which is of the Freundlich type [27].

\section{Acknowledgement}

The financial support of OTKA, Hungary (Grant No. $\mathrm{K} 120075$ ) is gratefully acknowledged. Z. Dorkó acknowledges the support of the Ernő Pungor Scholarship.

\section{References}

[1] Sellergren, B. (ed.) "Molecularly Imprinted Polymers: Man-Made Mimics of Antibodies and their Application in Analytical Chemistry." Techniques and Instrumentation in Analytical Chemistry, 1st ed., Elsevier Science, Amsterdam, 2001.

[2] Haupt, K. (ed.) "Molecular Imprinting." Springer, Heidelberg, 2012.

[3] Alvarez-Lorenzo, C., Concheiro, A. (eds.) "Handbook of Molecularly Imprinted Polymers." 1st ed., Smithers Rapra, Shrewsbury, 2013.

[4] Pap, T., Horvai, G. "Characterization of the selectivity of a phenytoin imprinted polymer." Journal of Chromatography A. 1034(1-2), pp. 99107. 2004. https://doi.org/10.1016/j.chroma.2004.01.064

[5] Foo, K. Y., Hameed, B. H. "Insights into the modeling of adsorption isotherm systems." Chemical Engineering Journal (Lausanne). 156(1), pp. 2-10. 2010. https://doi.org/10.1016/j.cej.2009.09.013

[6] Toth, J., Adsorption- Theory, Modeling and Analysis, 1st ed., Marcel Dekker, New York, 2002.
[7] Bellot, J. C., Condoret, J. S. "Modeling of liquid-chromatography equilibria." Process Biochemistry. 28(6), pp. 365-376. 1993. https://doi.org/10.1016/0032-9592(93)80023-A

[8] Alberti, G., Amendola, V., Pesavento, M., Biesuz, R. "Beyond the synthesis of novel solid phases: Review on modelling of sorption phenomena." Coordination Chemistry Reviews. 256(1-2), pp. 28-45. 2012. https://doi.org/10.1016/j.ccr.2011.08.022

[9] Guiochon, G., Felinger, A., Shirazi, D. G., Katti, A. M. "Fundamentals of Preparative and Nonlinear Chromatography." 2nd ed., Academic Press, Boston, 2006.

[10] Helfferich, F. "Ionenaustauscher. Band I: Grundlagen. Struktur- Herstellung-Theorie." Verlag Chemie, Weinheim, 1959. (in German)

[11] Abbate, V., Bassindale, A. R., Brandstadt, K. F., Taylor, P. G. "An artificial organosilicon receptor." Polymer Chemistry. 3(8), pp. 2018-2027. 2012. https://doi.org/10.1039/C2py20135f

[12] Tsermentseli, S. K., Manesiotis, P., Assimopoulou, A. N., Papageorgiou, V. P. "Molecularly imprinted polymers for the isolation of bioactive naphthoquinones from plant extracts." Journal of Chromatography A. 1315, pp. 15-20. 2013. https://doi.org/10.1016/j.chroma.2013.09.044

[13] Li, H., Nie, L. H., Yao, S. Z. "Adsorption isotherms and sites distribution of caffeic acid-imprinted polymer monolith from frontal analysis." Chromatographia. 60(7-8), pp. 425-431. 2004.

https://doi.org/10.1365/s10337-004-0403-9

[14] Schillinger, E., Moder, M., Olsson, G. D., Nicholls, I. A., Sellergren, B. "An artificial estrogen receptor through combinatorial imprinting." Chemistry-A European Journal. 18(46), pp. 14773-14783. 2012. https://doi.org/10.1002/chem.201201428

[15] Manesiotis, P., Kashani, S., McLoughlin, P. "Molecularly imprinted polymers for the extraction of imiquimod from biological samples using a template analogue strategy." Analytical Methods. 5(12), pp. 3122-3128. 2013. https://doi.org/10.1039/C3ay40239h

[16] Garcia-Calzon, J. A., Diaz-Garcia, M. E. "Characterization of binding sites in molecularly imprinted polymers." Sensors and Actuators B: Chemical. 123(2), pp. 1180-1194. 2007.

https://doi.org/10.1016/j.snb.2006.10.068

[17] Umpleby, R. J., Baxter, S. C., Rampey, A. M., Rushton, G. T., Chen, Y. Z., Shimizu, K. D. "Characterization of the heterogeneous binding site affinity distributions in molecularly imprinted polymers." Journal of Chromatography B. 804(1), pp. 141-149. 2004.

https://doi.org/10.1016/j.jchromb.2004.01.064

[18] Sajonz, P., Kele, M., Zhong, G. M., Sellergren, B., Guiochon, G. "Study of the thermodynamics and mass transfer kinetics of two enantiomers on a polymeric imprinted stationary phase." Journal of Chromatography A. 810(1-2), pp. 1-17. 1998. https://doi.org/10.1016/S0021-9673(98)00247-7

[19] Gritti, F., Guiochon, G. "Limits of the numerical estimation of the adsorption energy distribution from adsorption isotherm data using the expectation-maximization method." Journal of Chromatography A. 1144(2), pp. 208-220. 2007. https://doi.org/10.1016/j.chroma.2007.01.057

[20] Kong, S., Wang, Y., Hu, Q., Olusegun, A. "Magnetic nanoscale Fe-Mn binary oxides loaded zeolite for arsenic removal from synthetic groundwater." Colloids and Surfaces A: Physicochemical and Engineering Aspects. 457 pp. 220-227. 2014.

https://doi.org/10.1016/j.colsurfa.2014.05.066

[21] Podder, M. S., Majumder, C. B. "Sd/MnFe ${ }_{2} \mathrm{O}_{4}$ composite, a biosorbent for As(III) and As(V) removal from wastewater: Optimization and isotherm study." Journal of Molecular Liquids. 212, pp. 382-404. 2015. https://doi.org/10.1016/j.molliq.2015.09.011 
[22] Rey-Mafull, C. A., Tacoronte, J. E., Garcia, R., Tobella, J., Llopiz, J. C., Iglesias, A., Hotza, D. "Comparative study of the adsorption of acetaminophen on activated carbons in simulated gastric fluid." SpringerPlus. 3, p. 48. 2014. https://doi.org/10.1186/2193-1801-3-48

[23] Tomic, N. M., Dohcevic-Mitrovic, Z. D., Paunovic, N. M., Mijin, D. Z., Radic, N. D., Grbic, B. V., Askrabic, S. M., Babic, B. M., BajukBogdanovic, D. V. "Nanocrystalline $\mathrm{CeO}_{2-\delta}$ as effective adsorbent of azo dyes." Langmuir. 30(39), pp. 11582-11590. 2014.

https://doi.org/10.1021/la502969w

[24] Tan, J., Wei, X. Y., Ouyang, Y. X., Fan, J. H., Liu, R. "Adsorption properties of copper (II) ion from aqueous solution by starch-grafted polyacrylamide and crosslinked starch-grafted polyacrylamide." Periodica Polytechnica Chemical Engineering. 58(2), pp. 131-139. 2014.

https://doi.org/10.3311/PPch.7185
[25] Dorkó, Z., Tamás, B., Horvai, G. "Relationship between individual and competitive adsorption isotherms on molecularly imprinted polymers." Periodica Polytechnica Chemical Engineering. OnlineFirst (2017) paper 9726. https://doi.org/10.3311/PPch.9726

[26] Kamarei, F., Gritti, F., Guiochon, G., Burchell, J. "Accurate measurements of frontal analysis for the determination of adsorption isotherms in supercritical fluid chromatography." Journal of Chromatography A. 1329, pp. 71-77. 2014. https://doi.org/10.1016/j.chroma.2013.12.033

[27] Dorkó, Z., Szakolczai, A., Horvai, G., manuscript in preparation. 